\title{
Lymphatic vessels clean up your arteries
}

\author{
Carlos Fernández-Hernando
}

\author{
Departments of Medicine and Cell Biology, Leon H. Charney Division of Cardiology and the Marc and Ruti Bell Vascular Biology and Disease Program, \\ New York University School of Medicine, New York, New York, USA.
}

\begin{abstract}
Reverse cholesterol transport (RCT) is the pathway by which cholesterol accumulated in peripheral tissues, including the artery wall, is transported to the liver for excretion. There is strong evidence suggesting that interventions that increase macrophage cholesterol efflux and RCT would be antiatherogenic. In this issue of the JCI, Martel et al. investigate the contribution of lymphatic vasculature to RCT. Their results support the concept that the lymphatic vessel route is critical for RCT from atherosclerotic plaques. Therefore, strategies to improve lymphatic transport might be useful for treating atherosclerotic vascular disease.
\end{abstract}

HDL, reverse cholesterol transport, and coronary heart disease

A number of observational studies, including the Framingham Heart Study, have shown a strong inverse association of plasma HDL cholesterol levels with coronary heart disease (CHD) (1). HDL is thought to regulate RCT by promoting cholesterol efflux from arterial macrophage foam cells to the liver for biliary excretion, leading to a reduction in atherosclerotic plaque size $(2,3)$. Cholesterol efflux from macrophages is mediated primarily by the ATP-binding cassette transporters ABCA1 and ABCG1 (4). The expression level of these transporters is regulated at the transcriptional level by the liver $X$ receptor (LXR) nuclear hormone transcription factors and post-transcriptionally by miR-33, a microRNA encoded within intron 16 of the SREBP2 gene (Figure 1) $(5,6)$. Studies in mice have shown that activating LXR nuclear receptors or inhibiting miR-33 reduces the progression and enhances the regression of atherosclerosis (7-10). Altogether, these findings suggest that pharmacological interventions to increase macrophage cholesterol efflux in the arterial wall might be a promising strategy to prevent atherosclerosis.

Although observational, clinical, and preclinical studies all strongly suggest that plasma HDL cholesterol might be beneficial in preventing $\mathrm{CHD}$, three recent clinical trials (AIM-HIGH, dal-OUTCOMES, and HPS-THRIVE) that aimed to increase circulating HDL cholesterol levels were

Conflict of interest: The author has declared that no conflict of interest exists.

Citation for this article: J Clin Invest. 2013; 123(4):1417-1419. doi:10.1172/JCI68657. prematurely terminated on the basis of futility $(11,12)$. Moreover, a recent genetic study found that plasma HDL cholesterol levels had a weak or nonexistent relationship with CHD (13). These results suggest that HDL cholesterol levels are not per se an accurate indicator of CHD risk, and furthermore, that raising HDL cholesterol may not lead to an increased RCT capacity or to a reduction in cardiovascular events. To assess whether the capacity of HDL to promote cholesterol efflux from macrophages would serve as a better predictor of atherosclerotic burden, Rader and colleagues measured the cholesterol efflux capacity in apolipoprotein B-depleted serum (which is enriched in HDL particles) from CHD patients and controls (2). They showed that the capacity of HDL to promote macrophage cholesterol efflux was statistically associated with CHD risk, suggesting that therapeutic approaches aimed at enhancing HDL-mediated cholesterol efflux are likely to be effective in preventing CHD, regardless of their effects on circulating HDL cholesterol levels.

\section{Physiological role of lymphatic circulation on RCT}

Many aspects of the molecular mechanisms that regulate RCT remain unclear. For instance, how HDL particles interact with macrophages accumulated in atherosclerotic plaques and then leave the lesion after removing the excess cholesterol is not completely understood. In this issue of the JCI, Martel et al. provide intriguing findings which suggest that the lymphatic vasculature plays an essential role in regulating RCT (14). To explore the potential physiological relevance of the lymphatic circulation in removing cholesterol from peripheral tissues, the authors used two different models of disrupted lymphatic drainage from skin. In the first approach, they surgically separated dermal lymphatic vessels to disrupt lymph flow from the tail dermis of mice without damaging the blood circulation and injected $\left[{ }^{3} \mathrm{H}\right]$-cholesterol-loaded macrophages into the tail upstream of the lymphatic separation. Because RCT measurements using this technique in wild-type mice were near the lower limit of detection, the authors used Apoa1 transgenic mice, which have higher plasma HDL cholesterol and particle levels. They found that the surgical separation of lymphatic vessels in the tail significantly reduced RCT without affecting the plasma lipoprotein profile or macrophage cholesterol efflux capacity (14). To confirm these observations, the authors also used a mutant mouse model that lacks the expression of one allele of the vascular endothelial growth factor-C receptor (VEGFR3) (15). These mice, known as "Chy mice," selectively lose the lymphatic vasculature in the skin capillaries, but the body trunk skin retains some lymphatic vasculature. Similar to the surgical model, RCT in Chy mice was significantly impaired compared with controls (14).

Although these data strongly suggest that lymphatic vessels are critical to RCT, the molecular mechanism by which HDL leaves the tissue and returns to the bloodstream might be different in the skin compared with the artery wall. To address this issue, the authors turned to a very elegant and challenging model previously described by Fisher and colleagues (16), in which the aortic arch from an Apoe ${ }^{-/-}$ mouse (with established atherosclerosis) is transplanted into an $A p o e^{-/-}$mouse. In this approach, the surgery itself disrupts the lymphatic connections in the vessel wall after transplantation and prevents atherosclerotic plaque regression $\left(\mathrm{Apoe}^{-/-}\right.$ mice have elevated plasma cholesterol levels). To avoid reestablishing the lymphatic vessels after surgery, the authors treated a subset of the mice with neutralizing antiVEGFR3 mAb. Two weeks after transplantation and treatment with the antibody, 


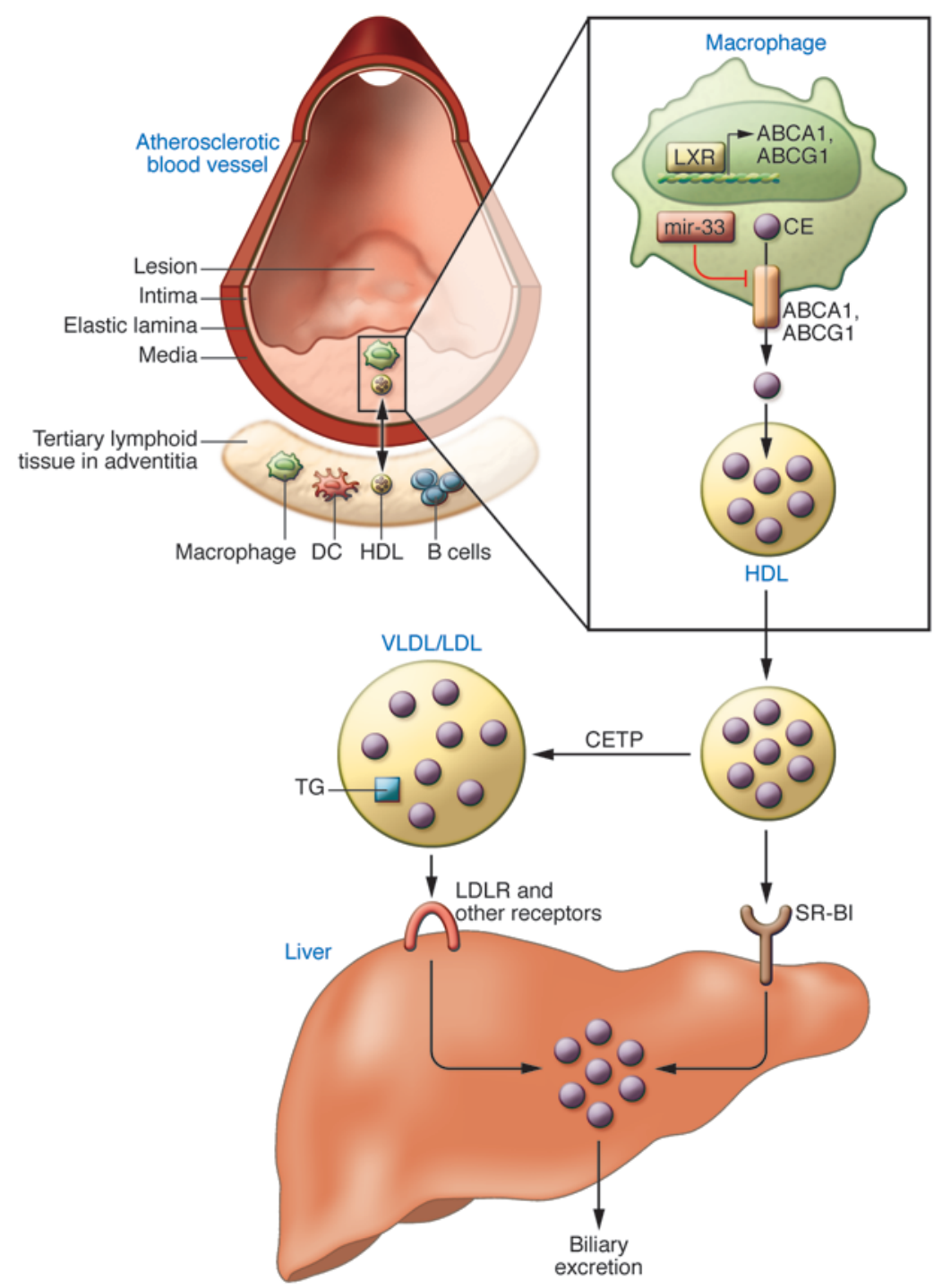

\section{Figure 1}

Regulation of RCT by HDL. The macrophages that accumulate in atherosclerotic plaques efflux cholesterol to HDL particles via ABCA1 and ABCG1. Both transporters are regulated at the transcriptional level by LXRs and post-transcriptionally by miR-33. HDL particles exit the artery wall and return to the liver via the SR-BI receptor. In the liver, most cholesterol is transformed into bile acids and is secreted into the bile for excretion. Cholesterol esters (CE) from HDL can also be transferred to VLDL/LDL by the cholesterol ester transfer protein (CETP), and after that can be removed from the plasma through the hepatic LDL receptor (LDLr). In this issue of the $J C l$, Martel et al. suggest that lymphatic vessels play a key role in controlling HDL particle entry into and exit from atherosclerotic lesions, thereby regulating RCT. TG, triglycerides.

mice were injected with an adenoviral vector encoding Apoe to improve the plasma lipoprotein profile (i.e., lower non-HDL$\mathrm{C}$, raise HDL-C) in order to promote cellular cholesterol efflux from atherosclerotic plaque macrophages. Remarkably, Martel et al. found that inhibition of lymphatic vessel regrowth impaired RCT and caused cholesterol accumulation in the transplanted aortae (14). Taken together, these findings strongly support the concept that lymphatic circulation plays a critical role in regulating RCT (Figure 1 ).

\section{Unanswered questions}

Important questions remain to be addressed to fully understand the role of lymphatic vessels in this process. For instance, the morphology of atherosclerotic plaques after treatment needs to be evaluated to assess whether the changes in cholesterol content in the transplanted arches are due to an increase in macrophage cholesterol efflux or to the lymphatic circulation allowing macrophage egress in this model. Indeed, previous data from the Randolph and Fisher laboratories have shown significant macrophage egress from the intima to the lymphatic vessels during regression in this transplant model (17). In addition, this model may not recapitulate the situation of the intact artery because the surgical intervention alters the medial immunoprivilege, leading to an increase in inflammation and lymphatic vessel development. In fact, while macrophage egress is quantitatively significant in the surgical model, it appears to be marginal in the model of regression achieved in Apoe ${ }^{-/-}$mice by ApoE complementation (18). Thus, further experiments using nonsurgical models will be important to determine the contribution of lymphatic vessels in RCT.

\section{Concluding remarks and future directions}

The lymphatic system is found in most tissues of the body and plays an important role in lipid transport from the intestine to the circulation (19). Most dietary lipids are absorbed in the small intestine and packaged in triglyceride-rich particles known as chylomicrons, and are then transported to the blood stream through the lymphatic system. Impaired lymphatic drainage in mice results in lipid accumulation in the interstitium (20). Similarly, patients with lymphedema appear to have compromised lipid transport and accumulate large lipid deposits. Some reports have also shown that several lipoprotein fractions are present in the lymph, including HDL. Indeed, the concentration of HDL cholesterol in the lymph is $30 \%$ greater than in the blood, suggesting a key role of lymphatic circulation in regulating RCT (21). In the present study, Martel et al. provide definitive evidence that impairment of lymphatic circulation compromises RCT from peripheral tissues, including the skin and a transplanted aortic vessel (14). It would be interesting to further assess whether the patients with lymphedema or those who undergo surgery or node removal have RCT defects, lower circulating HDL cholesterol, and increased risk of CHD. Finally, this study suggests that therapeutic interventions to improve lymphatic circulation might enhance lipid clearance from the artery wall and prevent atherosclerotic vascular disease. 


\section{Acknowledgments}

The Fernández-Hernando Lab is supported by grants from the NIH (R01HL107953 and R01HL106063).

Address correspondence to: Carlos Fernández-Hernando, 522 First Avenue, Smilow 703, New York, New York 10016, USA. Phone: 212.263.4122; Fax: 212.263.4129; E-mail: carlos.fernandez-hernando@ nyumc.org.

1. Castelli WP, et al. HDL cholesterol and other lipids in coronary heart disease. The cooperative lipoprotein phenotyping study. Circulation. 1977; 55(5):767-772.

2. Khera AV, et al. Cholesterol efflux capacity, high-density lipoprotein function, and atherosclerosis. N Engl J Med. 2011;364(2):127-135.

3. Rader DJ, Alexander ET, Weibel GL, Billheimer J, Rothblat GH. The role of reverse cholesterol transport in animals and humans and relationship to atherosclerosis. J Lipid Res. 2009;50(suppl):S189-S194.

4. Wang X, et al. Macrophage ABCA1 and ABCG1, but not SR-BI, promote macrophage reverse cholesterol transport in vivo. J Clin Invest. 2007;117(8):2216-2224.
5. Naik SU, et al. Pharmacological activation of liver $\mathrm{X}$ receptors promotes reverse cholesterol transport in vivo. Circulation. 2006;113(1):90-97.

6. Rayner KJ, et al. MiR-33 contributes to the regulation of cholesterol homeostasis. Science. 2010; 328(5985):1570-1573.

7. Feig JE, et al. LXR promotes the maximal egress of monocyte-derived cells from mouse aortic plaques during atherosclerosis regression. J Clin Invest. 2010; 120(12):4415-4424

8. Joseph SB, et al. Synthetic LXR ligand inhibits the development of atherosclerosis in mice. Proc Natl Acad Sci U S A. 2002;99(11):7604-7609.

9. Rayner KJ, et al. Antagonism of miR-33 in mice promotes reverse cholesterol transport and regression of atherosclerosis. J Clin Invest. 2011;121(7):2921-2931.

10. Verschuren L, et al. LXR agonist suppresses atherosclerotic lesion growth and promotes lesion regression in apoE*3Leiden mice: time course and mechanisms. J Lipid Res. 2009;50(2):301-311.

11. Boden WE, et al. Niacin in patients with low HDL cholesterol levels receiving intensive statin therapy. NEngl J Med. 2011;365(24):2255-2267.

12. Schwartz GG, et al. Rationale and design of the dal-OUTCOMES trial: efficacy and safety of dalcetrapib in patients with recent acute coronary syndrome. Am Heart J. 2009;158(6):896-901 e893.

13. Voight BF, et al. Plasma HDL cholesterol and risk of myocardial infarction: a mendelian randomisation study. Lancet. 2012;380(9841):572-580.
14. Martel C, et al. Lymphatic vasculature mediates macrophage reverse cholesterol transport in mice. J Clin Invest. 2013;123(4):1571-1579.

15. Karkkainen MJ, et al. A model for gene therapy of human hereditary lymphedema. Proc Natl Acad Sci US A. 2001;98(22):12677-12682.

16. Reis ED, et al. Dramatic remodeling of advanced atherosclerotic plaques of the apolipoprotein E-deficient mouse in a novel transplantation model. J Vasc Surg. 2001;34(3):541-547.

17. Llodra J, Angeli V, Liu J, Trogan E, Fisher EA, Randolph GJ. Emigration of monocyte-derived cells from atherosclerotic lesions characterizes regressive, but not progressive, plaques. Proc Natl Acad Sci US A. 2004;101(32):11779-11784.

18. Potteaux S, et al. Suppressed monocyte recruitment drives macrophage removal from atherosclerotic plaques of $\mathrm{Apoe}^{-/-}$mice during disease regression. J Clin Invest. 2011;121(5):2025-2036.

19. Iqbal J, Hussain MM. Intestinal lipid absorption. Am JPhysiol Endocrinol Metab. 2009;296(6):E1183-E1194.

20. Harvey NL, et al. Lymphatic vascular defects promoted by Prox 1 haploinsufficiency cause adult-onset obesity. Nat Genet. 2005;37(10):1072-1081.

21. Nanjee MN, Cooke CJ, Wong JS, Hamilton RL, Olszewski WL, Miller NE. Composition and ultrastructure of size subclasses of normal human peripheral lymph lipoproteins: quantification of cholesterol uptake by HDL in tissue fluids. J Lipid Res. 2001;42(4):639-648

\title{
Desmoglein-1, differentiation, and disease
}

\author{
Christoph M. Hammers and John R. Stanley
}

Department of Dermatology, University of Pennsylvania, Philadelphia, Pennsylvania, USA.

\begin{abstract}
Desmoglein-1 (DSG1), a desmosomal protein, maintains the structure of epidermis through its adhesive function. However, heterozygous mutations in DSG1 in humans result in abnormal differentiation, as does downregulation of DSG1 in human skin organ culture, suggesting that it may have important signaling functions. In this issue of the JCI, Harmon et al. elucidate how the binding of the DSG1 cytoplasmic tail to the scaffolding protein Erbin decreases signaling through the Ras-Raf pathway to promote stratification and differentiation of keratinocytes in the epidermis.
\end{abstract}

\section{Introduction}

In an era of research budget cuts and tightened paylines, the significance and translational potential of grant proposals are sometimes equated with the prevalence of the disease being studied and/or the obvious clinical application of the proposal. However, it is important to remember that we have learned a tremendous amount about the normal physiology and pathophysiology of organs through the study of rare diseases and through studies of the basic cell biology of seemingly boring (that is, uncommunicative) struc-

Conflict of interest: The authors have declared that no conflict of interest exists.

Citation for this article: J Clin Invest. 2013; 123(4):1419-1422. doi:10.1172/JCI69071. tural molecules. In this Commentary, we will illustrate this observation by tracing recent studies centered on desmoglein-1 (DSG1) and its associated diseases.

\section{The discovery of DSG1}

The family of desmogleins was characterized in the early 1990s through biochemical isolation, monoclonal antibody production, and then expression cloning, which showed they were in the supergene family of cadherins. Because desmogleins were present in the electron lucent center of the desmosome, a cell adhesion structure, and were in the cadherin family of adhesion molecules, they were presumed to function in cell-cell adhesion (1). In fact, "desmoglein" is composed of the Greek words "desmos" for "tie" and "glein" for "glue-like." Whereas DSG2 was found in all desmosome-possessing tissues, including nonepithelial myocardium, expression of the family members DSG1 and DSG3 was mainly limited to the epidermis and mucosa (2).

Molecular cloning also indicated that the desmoglein cytoplasmic tail had one segment with homology to that of classical cadherins. This segment binds the armadillo protein plakoglobin, an interaction thought to be important in desmosome assembly through cobinding of plakoglobin (and perhaps other armadillo proteins) to desmoplakin, the major desmosome plaque protein (refs. 3-5 and Figure 1). Desmogleins also have unique intracellular segments (6), the functions of which have now been partially elucidated by Harmon et al. (7) in this issue of the JCI.

\section{The rare autoimmune blistering skin disease pemphigus foliaceus elucidates the adhesive function of DSG1}

At about the same time that desmogleins were being characterized, the antigen of a rare autoimmune blistering skin disease, pemphigus foliaceus (PF), was 